\title{
WEAK TOTAL RESOLVABILITY IN GRAPHS
}

\author{
${\text { Katrin } \text { Casel }^{1} \text {, Alejandro Estrada-Moreno }}^{2}$, Henning Fernau $^{1}$ \\ AND \\ Juan Alberto Rodríguez-Velázquez ${ }^{2}$ \\ ${ }^{1} \mathrm{FB}$ 4-Abteilung Informatikwissenschaften \\ Universität Trier, 54286 Trier, Germany \\ ${ }^{2}$ Departament d'Enginyeria Informàtica i Matemàtiques \\ Universitat Rovira $i$ Virgili \\ Av. Països Catalans 26, 43007 Tarragona, Spain \\ e-mail: casel@informatik.uni-trier.de \\ alejandro.estrada@urv.cat \\ fernau@uni-trier.de \\ juanalberto.rodriguez@urv.cat
}

\begin{abstract}
A vertex $v \in V(G)$ is said to distinguish two vertices $x, y \in V(G)$ of a graph $G$ if the distance from $v$ to $x$ is different from the distance from $v$ to $y$. A set $W \subseteq V(G)$ is a total resolving set for a graph $G$ if for every pair of vertices $x, y \in V(G)$, there exists some vertex $w \in W-\{x, y\}$ which distinguishes $x$ and $y$, while $W$ is a weak total resolving set if for every $x \in V(G)-W$ and $y \in W$, there exists some $w \in W-\{y\}$ which distinguishes $x$ and $y$. A weak total resolving set of minimum cardinality is called a weak total metric basis of $G$ and its cardinality the weak total metric dimension of $G$. Our main contributions are the following ones: (a) Graphs with small and large weak total metric bases are characterised. (b) We explore the (tight) relation to independent 2-domination. (c) We introduce a new graph parameter, called weak total adjacency dimension and present results that are analogous to those presented for weak total dimension. (d) For trees, we derive a characterisation of the weak total (adjacency) metric dimension. Also, exact figures for our parameters are presented for (generalised) fans and wheels. (e) We show that for Cartesian product graphs, the weak total (adjacency) metric dimension is usually pretty small. (f) The weak total (adjacency) dimension is studied for lexicographic products of graphs.
\end{abstract}

Keywords: metric dimension, resolving set, weak total metric dimension, weak total resolving set, adjacency dimension, graph operations.

2010 Mathematics Subject Classification: 05C12, 05C76. 


\section{REFERENCES}

[1] R.C. Brigham, G. Chartrand, R.D. Dutton and P. Zhang, Resolving domination in graphs, Math. Bohem. 128 (2003) 25-36.

[2] G. Chartrand, V. Saenpholphat and P. Zhang, The independent resolving number of a graph, Math. Bohem. 128 (2003) 379-393.

[3] R. Hammack, W. Imrich and S. Klavžar, Handbook of Product Graphs, 2nd Ed. (CRC Press, 2011).

[4] F. Harary and R.A. Melter, On the metric dimension of a graph, Ars Combin. 2 (1976) 191-195.

[5] T.W. Haynes, S.T. Hedetniemi and P.J. Slater, Fundamentals of Domination in Graphs (Chapman and Hall/CRC Pure and Applied Mathematics Series, Marcel Dekker, Inc. New York, 1998).

[6] I. Javaid, F. Iftikhar and M. Salman, Total resolvability in graphs, Middle-East Journal of Scientific Research 11 (2012) 1649-1658.

[7] F. Okamoto, B. Phinezy and P. Zhang, The local metric dimension of a graph, Math. Bohem. 135 (2010) 239-255.

[8] A. Sebö and E. Tannier, On metric generators of graphs, Math. Oper. Res. 29 (2004) 383-393.

doi:10.1287/moor.1030.0070

[9] P.J. Slater, Leaves of trees, Congr. Numer. 14 (1975) 549-559.

Received 20 October 2014

Revised 29 May 2015

Accepted 29 May 2015 